\title{
Influência do treinamento de força muscular e de flexibilidade articular sobre 0 equilíbrio corporal em idosas
}

\author{
Influence of strengthtraining andflexibility on boody balancein eldely
}

Introdução: $\mathrm{O}$ aumento da proporção de idosos na população mundial traz à tona a discussão a respeito de eventos incapacitantes nessa faixa etária, entre os quais se destaca a ocorrência de quedas. A redução da flexibilidade articular e da força muscular são as principais variáveis motoras relacionadas às maiores limitações das atividades de vida diária (AVDs) e aos altos índices de quedas registrados na população idosa. Objetivo: este estudo se propõe a verificar a influência do treinamento de força muscular e de flexibilidade articular sobre o equilíbrio corporal em idosas. Metodologia: A amostra foi composta por 22 mulheres, de 60 a 75 anos, participantes de dois programas de atividade física. Sete sujeitos participaram do programa de força e 15 fizeram parte do programa de flexibilidade. O equilíbrio foi avaliado antes e após o período de treinamento (Escala de Berg). Resultados: constatou-se que, em ambos os grupos, o equilíbrio se mostrou significativamente maior após os programas de treinamento. No grupo que participou do treinamento de força, a média dos pontos pré e pós-treinamento foi de 53 e 55,86, respectivamente. No grupo que participou do treinamento de flexibilidade, as médias pré e pós foram 52,47 e 55,47 pontos, respectivamente. Conclusão: constatou-se que os dois treinamentos produziram melhoras nos índices de equilíbrio corporal de idosas, o que provavelmente poderá influenciar na redução da incidência de quedas e da perda da independência física, assim como na obtenção de melhor qualidade de vida.

Grêmio Náutico União. Sede Alto Petrópolis. Porto Alegre, RS, Brasil.

2 Centro de Desportos, Departamento de Educação Física. Universidade Federal de Santa Catarina. Florianópolis, SC, Brasil.

3 Instituto de Psicologia, Departamento de Psicologia do Desenvolvimento e da Personalidade. Universidade Federal do Rio Grande do Sul. Porto Alegre, RS, Brasil.

4 Escola de Educação Física, Departamento de Educação Física.Universidade Federal do Rio Grande do Sul. Porto Alegre, RS, Brasil.

5 Curso de Educação Física, Campus Canoas. Universidade Luterana do Brasil. Porto Alegre, RS, Brasil.

6 Instituto de Geriatria e Gerontologia, Programa de Pós-Graduação em Gerontologia Biomédica. Pontifícia Universidade Católica do Rio Grande do Sul. Porto Alegre, RS, Brasil.
Igna Luciara Raffaeli Albino' Cíntia de la Rocha Freitas ${ }^{2}$ Adriane Ribeiro Teixeira ${ }^{3}$ Andréa Krüger Gonçalves ${ }^{4}$ Ana Maria Pujol Vieira dos Santos ${ }^{5}$ Ângelo José Gonçalves Bós ${ }^{6}$
Palavras-chave: Idosos. Força muscular. Avaliação de desempenho. Equilibrio postural. Flexibilidade articular. 


\section{Abstract}

Introduction: Increasing proportion of elderly people in the world population raises the discussion of disabling events in this age group, as the occurrence of falls. Reduction in joint flexibility and muscle strength are the main variable related to the major motor limitations of activities of daily living (ADLs) and high rates of reported falls in elderly. Objective: This study aims to assess the influence of muscle strength training and flexibility training on body balance in elderly. Methodology: The sample was composed by 22 women, from 60 to 75 years old, enrolled in two physical activity programs. Seven subjects were submitted to muscle strength program and 15 subjects were submitted to flexibility training. Body balance was evaluated before and after practicing time (Berg Balance Scale). Results: It was found that both groups had significant improvement in body balance. Average scores in strength training group were 53 and 55.86 points, respectively, before and after training, while the average scores in flexibility group were 52.47 and 55.47 points, before and after training. Conclusion: It was found that both training improved the rates of body balance in elderly women, which likely will influence the reduction of incidence of falls and loss of physical independence, as well as better quality of life.
Key words: Elderly. Muscle strength . Performance appraisal. Postural balance. Flexibility.

\section{INTRODUÇÃOO}

O envelhecimento imprime alterações naturais em todo o organismo. O processo biológico se traduz pelo declínio harmônico de todo o conjunto orgânico. Progressiva atrofia muscular, fraqueza funcional, descalcificação óssea, aumento da espessura da parede de vasos, aumento do nível de gordura corporal total e diminuição da capacidade coordenativa são algumas das alterações morfológicas e fisiológicas, geradas durante o processo de envelhecimento. $\mathrm{O}$ idoso responde com mais lentidão e menos eficazmente às alterações ambientais, tornando-se mais vulnerável. ${ }^{1,2,3}$

Os efeitos deletérios na capacidade funcional e a deterioração da mobilidade ocorrem, com o avanço da idade, sobretudo pela perda gradativa da massa muscular e, consequentemente, da força muscular. A sarcopenia tem impacto significante na saúde pública, sobretudo na população idosa, por suas bem conhecidas consequências funcionais no andar e no equilíbrio, que aumentam o risco de queda e levam à perda da independência física funcional, contribuindo para a ampliação do risco de doenças crônicas como diabetes e osteoporose. ${ }^{4}$
Aproximadamente $30 \%$ dos indivíduos com mais de 65 anos de idade caem ao menos uma vez por ano. Em parte, as quedas ocorrem em função de limitações fisiológicas, perda de massa muscular, limitações cognitivas e uso de medicamentos, tais como os benzodiazepínicos. ${ }^{5}$ No Brasil, estudo demonstrou que as mulheres têm maior risco de quedas que os homens. ${ }^{6}$

Nos idosos, a elasticidade de tendões, ligamentos e cápsulas articulares diminui devido a deficiências no colágeno. ${ }^{7,8}$ Durante a vida ativa, indivíduos adultos perdem cerca de $8-10 \mathrm{~cm}$ de flexibilidade na região lombar e no quadril. Particularmente, a flexibilidade dos músculos isquiotibiais durante a senescência tem importante papel no equilíbrio postural, na manutenção completa da amplitude de movimento do joelho e quadril, na prevenção de lesões e na otimização da função músculo-esquelética. ${ }^{9}$

Em função destas limitações, tem sido apontado que a prática regular de exercício físico consiste em tratamento eficaz na melhora da capacidade funcional de indivíduos com idade avançada. ${ }^{10} \mathrm{~A}$ ação preventiva que o exercício físico produz tem importante implicação no que diz respeito à saúde, por reduzir a probabilidade de 
doenças, incapacidades e mortalidade desta população. ${ }^{11}$

A estimulação corporal global favorece o melhor desempenho das atividades diárias. Assim, a melhor opção para o indivíduo que está envelhecendo é a realização de um programa de atividade física que inclua treinamento aeróbico, força muscular, flexibilidade e equilíbrio. ${ }^{12} \mathrm{Nos}$ últimos anos, ficou provado que os idosos podem se beneficiar com a participação em programas de treinamento de força. Estudos comprovaram que até mesmo indivíduos com idade acima de 90 anos podem conseguir ganhos em força, durante um período treinamento de oito semanas. A síntese proteica em idosos ocorre mais lentamente do que em adultos jovens, mas a comparação de secções transversas de músculos entre pessoas ativas e inativas sugere que muito da perda de tecido magro pode ser evitada por um treinamento regular com pesos. Músculos preparados melhoram a função das articulações e reduzem o risco de quedas. ${ }^{13}$ Estudos evidenciaram maior tamanho das fibras musculares e maior força muscular em idosos em treinamento de força e em treinamento de resistência, quando comparados com o grupo controle. $^{14}$

Neste propósito, destaca-se a importância do desenvolvimento da capacidade motora do indivíduo que envelhece. Sabe-se que a perda $\mathrm{da}$ flexibilidade e da força são as principais variáveis motoras relacionadas às maiores limitações das atividades de vida diária (AVDs) e aos altos índices de quedas, pela diminuição ou perda da mobilidade, registrados nesta população. ${ }^{15}$

O objetivo deste estudo foi verificar a influência de um treinamento de força muscular e de um treinamento de flexibilidade articular sobre o equilíbrio corporal de idosos.

\section{METODOLOGIA}

A amostra foi composta por 22 indivíduos do sexo feminino, com idade entre 60 e 75 anos, participantes de programas de atividade física de um centro municipal da cidade de Porto Alegre (Ginásio Tesourinha), sendo sete frequentadoras do programa de força muscular $(65,1 \pm 2,9$ anos) e 15 frequentadoras do programa de flexibilidade (66,5 $\pm 3,2$ anos), todas, em ambos os grupos, há mais de um ano. Todos os indivíduos foram escolhidos por conveniência. Considerou-se como critério de exclusão a história prévia de distúrbios vestibulares. Foi analisado também se as participantes da amostra praticavam outra atividade, além daquelas a que estariam submetidas no presente estudo (exercícios de força e de flexibilidade).

Constatou-se que, em ambos os grupos, todas eram praticantes da caminhada, de duas a três vezes por semana, por aproximadamente uma hora, há mais de um ano. Todas as voluntárias assinaram um termo de consentimento livre e informado, declarando que aceitavam participar da pesquisa. Para a coleta de dados, foi aplicada uma anamnese, elaborada especialmente para este estudo.

Posteriormente, as voluntárias foram instruídas sobre como seria o processo da testagem e informadas que, em alguns dos procedimentos de coleta dos dados, seria levado em conta o uso de apoio das mãos e o tempo para realizar os procedimentos. A seguir, foram combinados dias, horários e local para aplicação do teste, o qual seria realizado individualmente, antes $\mathrm{da}$ atividade física, e repetido 13 semanas após o treinamento (pré-teste e pós-teste).

O instrumento utilizado para o estudo foi o teste de mensuração do equilíbrio corporal (Escala de Equilíbrio de Berg $\left.{ }^{16}\right)$. Durante a aplicação dessa escala, mantiveram-se sempre presentes dois professores responsáveis pela coleta dos dados, uma pessoa responsável pela aplicação e demonstração da testagem e outra para assessorar, caso ocorresse alguma eventualidade durante o processo. Todos os profissionais envolvidos na coleta de dados eram professores de Educação Física.

Após a primeira fase da coleta dos dados, todos os resultados foram digitados e armazenados, para serem comparados com os resultados a serem obtidos na segunda fase (pós-treinamento). 
Protocolos de Treinamento de Força

Muscular e de Flexibilidade Articular

O protocolo de treinamento foi constituído por um programa de treinamento físico durante 11 semanas, realizado com a frequência de duas sessões semanais, com duração de uma hora e meia, tanto para o de força muscular quanto para o de flexibilidade articular.

As participantes do grupo de treinamento de força realizaram exercícios de força muscular, que consistia em desempenhar três séries de dez repetições com cargas de aproximadamente $70 \% \mathrm{da}$ carga máxima (carga obtida através do método de tentativa e erro, que consiste em adicionar carga até que o indivíduo consiga realizar, no máximo, 10 repetições) dos exercícios puxada dorsal, voador invertido, voador, rosca direta, extensão de tríceps, pressão de pernas (leg press), cadeira extensora, mesa flexora, cadeira adutora, cadeira abdutora e flexão plantar, com repouso passivo de dois minutos entre as séries. Os músculos abdominais foram os únicos trabalhados com um método diferente, que consistia em executar maior número de repetições durante as séries, sem a utilização de cargas, com o objetivo de desenvolver a resistência muscular localizada..$^{1718}$

Os sujeitos foram orientados para que as cargas de treinamento fossem reajustadas sempre que o limite superior de repetições preestabelecido para cada exercício fosse facilmente atingido em todas as séries, no intuito de preservar a intensidade inicial. Em todos os reajustes ocorridos, um profissional de Educação Física se encarregava de garantir a segurança e a exatidão das sobrecargas.

As participantes do grupo do treinamento de flexibilidade realizaram inicialmente um trabalho com a utilização de bolinhas de tênis, para o distensionamento da musculatura da base plantar dos pés, com movimentos de rolamento e pressão plantar contra a bolinha por toda a extensão do pé. O objetivo deste trabalho inicial foi aumentar a base plantar de contato com o chão, para propiciar maior estabilidade corporal. ${ }^{19}$

Após o trabalho inicial, visando à melhora do equilíbrio corporal, as idosas passaram a realizar os exercícios específicos de flexibilidade articular predeterminados (os exercícios específicos de flexibilidade articular aplicados foram selecionados após a realização de pesquisa sobre quais exercícios de flexibilidade e quais articulações seriam mais enfatizadas, levando em consideração o grau de envolvimento com controle do equilíbrio corporal). Exercitaram-se todas as principais articulações e grupos musculares envolvidos (articulações: cervical, ombro, punho, lombar, quadril, joelho, tornozelo). Os exercícios foram ministrados em maior proporção para as articulações de membros inferiores; com utilização de apoio bipodal e unipodal; com e sem utilização da visão. A duração de cada exercício de sustentação foi de 20 a 30 segundos. Os exercícios que envolveram deslocamento corporal de determinada posição inicial com o objetivo de atingir outra posição final, na qual era realizada a sustentação, foram repetidos, no mínimo, três vezes cada. ${ }^{18}$

Os vinte minutos finais de ambas as sessões de treinamento foram utilizados para realizar o relaxamento corporal. As sessões de treinamento tiveram a supervisão de profissionais $\mathrm{da}$ área de Educação Física.

As participantes da pesquisa foram informadas que, durante o período de intervenção, só poderiam realizar a caminhada como atividade física extra, além do programa de treinamento de força muscular ou de flexibilidade articular, e que deveriam ter, no mínimo, $80 \%$ de frequência durante o período de treinamento, caso contrário, seriam excluídas do estudo.

O projeto foi elaborado e desenvolvido de acordo com as diretrizese normas regulamentadoras de pesquisa envolvendo seres humanos e foi aprovado pelo Comitê de Ética em Pesquisa da Universidade Luterana do Brasil (Protocolo $\mathrm{n}^{\circ}$ 0599). Todos os sujeitos da amostra foram esclarecidos sobre o estudo e assinaram o Termo de Consentimento Livre e Esclarecido.

\section{Análise de dados}

Para o tratamento estatístico dos dados, utilizou-se o pacote estatístico SPSS (Statistical Packagefor the Social Sciences), versão 10.0. 
Utilizou-se inicialmente o teste KolmogorovSmirnov para verificar a normalidade dos dados. Este teste apontou que os dados relativos ao grupo submetido ao treinamento de força estavam dentro da normalidade e os dados relativos ao grupo submetido ao treinamento de flexibilidade não estavam dentro da normalidade. Sendo assim, utilizou-se um teste paramétrico, o teste $t$ de Student pareado, para comparar o equilíbrio corporal entre o pré e pós-teste do grupo submetido ao treinamento de força muscular. O teste não paramétrico de Wilcoxon foi utilizado para comparar o equilíbrio corporal entre o pré e pós-teste do grupo submetido ao treinamento de flexibilidade. Foi considerado um nível de significância de 0,05 $(P<0,05)$.

\section{RESULTADOS}

Os resultados do estudo apontam que tanto o treinamento de flexibilidade articular, quanto o de força muscular, desenvolvidos durante 11 semanas, resultaram em aumento do equilíbrio corporal das participantes.

Os resultados do grupo submetido ao treinamento de força muscular são apresentados na tabela 1. A média do escore da Escala de Berg das participantes que foram submetidas ao treinamento de força muscular foi $53( \pm 1,82)$ pontos no pré-teste e de $55,85( \pm 0,37)$ no pósteste. Houve também aumento significativo no índice de força muscular, após o período de treinamento $(p=0,003)$.

Tabela 1 - Resultados do pré e do pós-teste de equilíbrio corporal dos sujeitos do grupo submetido ao treinamento de força (pontos na Escala de Berg). * indica p < 0,05. Porto Alegre, RS, 2010.

\begin{tabular}{ccc}
\hline Sujeitos & Pré-teste & Pós-teste \\
\hline 1 & 55 & 56 \\
2 & 52 & 56 \\
3 & 53 & 56 \\
4 & 55 & 56 \\
5 & 52 & 56 \\
6 & 50 & 55 \\
7 & 54 & 56 \\
\hline Média & 53 & $55,85714^{*}$ \\
DP & 1,825742 & 0,377964 \\
\hline
\end{tabular}

A tabela 2 mostra os resultados do grupo de sujeitos que participou do treinamento de flexibilidade. A média do escore da Escala de Berg das participantes que foram submetidas ao treinamento de flexibilidade foi $52,46( \pm 1,72)$ pontos no pré-teste e de 55,46 $( \pm 0,74)$ no pósteste. Observa-se que este grupo também apresentou aumento significativo no índice de equilíbrio corporal, após o período de treinamento $(p=0,001)$. 
Tabela 2 - Resultados do pré e do pós-teste de equilíbrio corporal dos sujeitos do grupo submetido ao treinamento de flexibilidade (pontos na Escala de Berg). * indica p < 0,05. Porto Alegre, RS, 2010.

\begin{tabular}{ccc}
\hline Sujeitos & Pré-teste & Pós-teste \\
\hline 1 & 53 & 56 \\
2 & 52 & 55 \\
3 & 54 & 56 \\
4 & 51 & 55 \\
5 & 49 & 54 \\
6 & 51 & 55 \\
7 & 50 & 55 \\
8 & 53 & 56 \\
9 & 53 & 56 \\
10 & 53 & 56 \\
11 & 55 & 56 \\
12 & 52 & 56 \\
13 & 52 & 54 \\
14 & 54 & 56 \\
15 & 55 & 56 \\
Média & 52,46667 & $55,46667 *$ \\
DP & 1,726543 & 0,743223 \\
\hline
\end{tabular}

\section{DISCUSSÃO}

Tendo em vista que a força muscular e a flexibilidade, dentre outras valências físicas, interferem no equilíbrio corporal dos indivíduos idosos, ${ }^{15}$ esperava-se que o treinamento de 11 semanas, desenvolvido neste estudo, resultasse em melhora nos índices de equilíbrio corporal das idosas.

Uma série de estudos tem mostrado que os idosos sofrem redução do equilíbrio. ${ }^{20-23}$ Apesar de não ter havido grupo controle neste estudo, o que foi uma limitação, parece que a melhora do equilíbrio apresentada se deve à intervenção dos programas de exercício a que os sujeitos foram submetidos.

Durante o processo de envelhecimento humano, a força muscular tende a diminuir. Isto ocorre devido à diminuição da massa muscular magra (sarcopenia), acarretando o aumento da dependência funcional. ${ }^{7}$ As idosas que participaram do treinamento de força muscular neste estudo apresentaram melhora significativa em seu equilíbrio corporal (tabela 1), analisado pela Escala de Berg. ${ }^{16}$

O treinamento de força pode minimizar ou retardar o processo de sarcopenia para obter significantes respostas neuromusculares (hipertrofia muscular e força muscular), por meio do aumento da capacidade contrátil dos músculos esqueléticos. O treinamento de força tem sido apontado como fator atuante para evitar quedas nas situações de desequilíbrio do corpo, pelo aumento da massa magra e força muscular. ${ }^{24}$ Sugere-se que esse tipo de treinamento, por no mínimo duas vezes por semana, com cargas de aproximadamente $70 \%$ da carga máxima e com ênfase maior aos membros inferiores, produz melhoras significativas no equilíbrio corporal em idosos. 
Os incrementos de força são destacados de acordo com a intensidade dos exercícios. $\mathrm{O}$ treinamento de baixa intensidade em idosos reporta incrementos de força de menos de $20 \%$, enquanto o treinamento de força de moderada a alta intensidade resulta em incrementos acima de $227 \%$ de 1RM (repetição máxima), auxiliando na melhora de sua mobilidade funcional e na independência funcional. ${ }^{24}$

Sabe-se hoje que o treinamento de força de alta intensidade é seguro para esta população e produz aumentos significativos em força muscular, porém sem aumento relevante no tamanho do músculo. Ressalta-se que o aumento da força está associado ao aumento $d a$ velocidade $d a$ caminhada, à potência para subir escadas, ao equilíbrio e à atividade espontânea geral. ${ }^{8}$

O treinamento de força desenvolvido neste estudo, baseado nos princípios biomecânicos do controle corporal, ofereceu maior ênfase aos membros inferiores. ${ }^{25,26}$ Barela ${ }^{25} \mathrm{e}$ Freitas ${ }^{26}$ sugerem que, para manter o equilíbrio corporal, é necessário manter a projeção do centro de massa (CM) dentro da base de apoio (BA) e que, para isso ocorrer, é necessária determinada força muscular, obtida através das contrações musculares, a fim de se obter o controle da estabilidade ou do movimento do CM dentro da BA.

Para a ênfase atribuída aos membros inferiores, também influenciaram o aumento do tempo de reação do sistema nervoso central em atuar pelo uso de padrões de coordenação postural ou estratégias motoras, perante as perturbações corporais, e a perda progressiva da força muscular que, durante a senescência, ocorre em maior proporção nos membros inferiores do que nos superiores.

A diferença na força muscular entre membros inferiores e superiores advém possivelmente da maior diminuição do uso das pernas, com a idade. ${ }^{13} \mathrm{O}$ treinamento de força muscular destacase, pois, como primordial para a saúde durante o envelhecimento humano. Os resultados indicaram efeito positivo e significativo na melhora do equilíbrio corporal, mas o número reduzido de participantes no grupo de treinamento de força muscular (sete sujeitos) constitui uma limitação deste estudo.

Outra valência física, além da força muscular, que tende a interferir no equilíbrio corporal em indivíduos idosos, é a flexibilidade. Ela foi aqui analisada e apresentou diferença significativa. A restrição na amplitude de movimento das grandes articulações se torna maior no período da aposentadoria e, eventualmente, a independência é ameaçada, contribuindo assim para os altos índices de quedas associados aos idosos.

Os sujeitos que neste estudo participaram do treinamento da flexibilidade articular apresentaram melhora significativa em seu equilíbrio corporal, analisado pela Escala de Berg (tabela 2). Esses achados podem estar relacionados com a melhora das habilidades requeridas para a realização das atividades de vida diária.

Tomando como ponto de referência o treino, as atividades de distensão da base de apoio, a utilização ou não do sistema visual, assim como o uso de apoios bipedal e unipedal, tiveram como referencial as informações disponíveis na literatura. Um dos princípios biomecânicos que influenciam a estabilidade do corpo é a base de apoio que, na postura ereta, é representada pela área compreendida entre os calcanhares e os pés. Quanto maior for esta área, maiores serão os limites de estabilidade do sujeito e a área disponível para ele controlar seu centro de massa a fim de manter a estabilidade..$^{27,28}$ Desta forma, é de se esperar que qualquer redução dessa área afete o equilíbrio.

A utilização ou não do sistema visual teve como referência os estudos de Mochizuki. ${ }^{29} \mathrm{O}$ autor cita que a detecção de movimento através do sistema visual serve para perceber o movimento de objetos, manter o movimento ocular para a perseguição contínua de objetos e guiar o movimento corporal no ambiente. Desta maneira, ao realizar os exercícios com os olhos fechados, não se obtém a informação do movimento corporal em relação a um objeto ou ambiente para gerar respostas adequadas para garantir a 
estabilidade corporal, exigindo maior grau de controle corporal.

A utilização de apoio bipodal e unipodal teve como referência pesquisas de Teodori, Guirro \& Santos. ${ }^{19}$ Eles destacam que a assimetria na distribuição de peso na planta dos pés, mantida por algum tempo, acarreta a desorganização postural geral, havendo sobrecarga de alguns grupos musculares em detrimento de outros. Nesse sentido, evidencia-se a importância da intervenção corretiva, baseada na reorganização das atitudes posturais anormais por meio de reequilíbrio muscular, recuperando-se o comprimento e a flexibilidade musculares.

Assim como no treinamento de força muscular, no treinamento de flexibilidade articular foi atribuída maior ênfase aos membros inferiores. Os motivos para isto são os mesmos referidos no treinamento de força. Como ao se envelhecer ocorrem alterações fisiológicas e morfológicas no organismo humano, automaticamente a função muscular de contrair e alongar também se altera.

Durante a vida ativa, indivíduos adultos perdem cerca de $8-10 \mathrm{~cm}$ de flexibilidade na região lombar e no quadril, devido à diminuição da elasticidade de tendões, ligamentos e cápsulas articulares por causa da deficiência de colágeno. ${ }^{7,8}$ Neste estudo, os indivíduos testados (em ambos os treinamentos) apresentaram melhora significativa no equilíbrio mensurado pela Escala

\section{REFERÊNCIAS}

1. Farinatti PTV. Teorias biológicas do envelhecimento: do genético ao estocástico. Rev Bras Med Esporte São Paulo 2002 ago; 8(4): 129-38.

2. Kuhnen AP, et al. Programa de atividade física para terceira idade do CDS/UFSC: o efeito do exercício físico na resistência muscular. Extensio: Rev Eletrônica de Extensão Florianópolis 2004 mai; 1.

3. Hayflick L. Como e porque envelhecemos. Rio de Janeiro: Campus; 1997. 366p.

4. Matsudo SM et al. Evolução do Perfil Neuromotor e Capacidade Funcional de de Berg. Quando o escore da Escala de Berg está entre 43 a 52, cada ponto diminuído no escore equivale a 2 a $7 \%$ de aumento de risco de queda em idosos. ${ }^{30}$ Infere-se, portanto, que tendo as participantes obtido aumento significativo deste escore, sua probabilidade de quedas se torna menor e o grau de controle corporal, maior.

\section{CONCLUSÕES}

Os programas de treinamento empregados neste estudo mostraram que tanto o de força muscular, quanto o de flexibilidade articular, durante 11 semanas, foram eficientes para gerar benefícios em relação ao equilíbrio corporal das idosas avaliadas.

Com os dados significativos obtidos nos treinamentos realizados, constata-se que a melhora ou manutenção da força muscular e flexibilidade articular tem grande influência no equilíbrio corporal em idosos, podendo reduzir o risco de quedas e melhorar a qualidade de vida. Com isso, prolonga-se o período de independência, tão importante em qualquer idade, mas principalmente no período da senescência.

Sugere-se que novos estudos sejam realizados por períodos de tempo mais prolongados, envolvendo indivíduos de ambos os sexos e utilizando programas mistos, que enfoquem tanto o desenvolvimento da força muscular, quanto da flexibilidade.
Mulheres Fisicamente Ativas de acordo com a Idade Cronológica. Rev Bras Med Esporte São Paulo 2003 nov/dez; 9(6): 365-76.

5. Coutinho E, et al. Risk factors for falls with severe fracture in elderly people living in a middle-income country: a case control study. BMC Geriatrics 2008 aug; 8:21.

6. Pinheiro MM, et al. Risk factors for recurrent falls among brazilian women and men: the brazilian osteoporosis Study (BRASOS). Cad Saúde Pública 2010 janeiro; 26(1): 89-96. 
7. Rebelatto Jr, et al. Influência de um programa de atividade física de longa duração sobre a força muscular manual e a flexibilidade corporal de mulheres idosas. Rev Bras Fisioter 2006 jan; 10(1):127-32.

8. Fleck SJ, Kraemer WJ. Fundamentos do treinamento de força muscular. 2 ed. Porto Alegre: Artmed; 1999. 376p.

9. Brasileiro JS. Influência do resfriamento e do aquecimento local na flexibilidade dos músculos isquiotibiais. Rev Bras Fisioter 2007 janeiro;11(1): 57-61.

10. Borges MRD, Moreira AK. Influência da prática de atividades físicas na terceira idade: estudo comparativo dos níveis de autonomia para o desempenho nas AVDs e AIVDs entre idosos fisicamente ativos e idosos sedentários. Motriz Rio Claro 2009 jul/set; 15(3): 562-73.

11. Lima RM, Oliveira RJ, Silva VAP. Efeitos do treinamento resistido sobre a capacidade cardiorrespiratória de indivíduos idosos. Rev Digital Buenos Aires 2003 may; 10(84). [Acesso em 27 jun 2010]. Disponível em: http:// www.efdeportes.com/efd84/idosos.htm.

12. Matsudo SM. Atividade física na promoção da saúde e qualidade de vida no envelhecimento. Rev Bras Ed Física Especial São Paulo 2006 set; 20(5): 135-37.

13. Shepard, RJ. Aging and exercise. Internet society sport science 1998;3(4). Disponível em: http://sportsci.org. 1998.

14. Aagard P, et al. Mechanical muscle function, morphology, and fiber type in lifelong trained elderly. Med Sci Sports Exerc Indianapolis 2007 nov; 39(11): 1989-96.

15. Jacob Filho, W. Atividade física e envelhecimento saudável. Rev Bras Ed Física e Esporte 2006 set; 20(5): 73-7.

16. Miyamotto STT, et al. Brazilian Version of the Berg Balance Scale. Braz J Med Biol Res 2004 apr; 37(9): 1411-21.

17. Raso V, Matsudo SM, Matsudo VKR. A Força muscular de mulheres idosas decresce principalmente após oito semanas de interrupção de um programa de exercícios com pesos livres. Rev Bras Med Esporte $2001 \mathrm{dez}$; 7(5): 177-86.

18. American College os Sports Medicine. Diretrizes do ACSM para os testes de esforço e sua prescrição. 6 ed. Rio de Janeiro: Guanabara Koogan; 2003. 260p.

19. Teodori RM, Guirro EC, Santos RM. Distribuição da pressão plantar e localização do centro de força após a intervenção pelo método de reeducação postural global: um estudo de caso. Fisioter Mov 2005 jan/mar; 18(1): 27-35.

20. Manchester D, et al. Visual, vestibular and somatosensory contributions to balance control in the older adult. J Gerontol A Biol Sci Med Sci Oxford 1989 jul; 44(4): 18-127.

21. Lord SR, Ward JA. Age-associated differences in sensori-motor function and balance in community dwelling women. Age Ageing Oxford 1994 nov; 23: 452-60.

22. Maki BE, Holliday PJ, Topper AK. A prospective study of postural balance and risk of falling in an ambulatory and independent elderly population. J Gerontol Biol Sci Med Sci Oxford 1994 march; 49(2):72-84.

23. Choy NL, Brauer S, Nitz J. Changes in postural stability in women aged 20 to 80 years. J Gerontol A Biol Sci Med Sci Oxford 2003 Jun; 58(6): M525-M530.

24. Maior AS. Relação sarcopenia e treinamento de força. Rev Fisioter UNICID São Paulo 2004 jul/ dez; 3(2):125-39.

25. Barela JA. Estratégias de controle em movimentos complexos: ciclo percepção-ação no controle postural. Rev Paul Educ Fís 2000; sup.3:79-88.

26. Freitas SMS. Coordenação postural em adultos e idosos durante movimentos voluntários na postura ereta. São Paulo. Tese [Doutorado em Educação física] - Universidade de São Paulo; 2005.

27. Freitas Jr PB. Características comportamentais do controle postural de jovens, adultos e idosos . São Paulo. Dissertação[Mestrado em Biociências] - Universidade Estadual Paulista; 2003.

28. Okuno E, Fratin L. Desvendando a física do corpo humano: biomecânica. São Paulo: Manole; 2003. 200p.

29. Mochizuki L, Amadio AC. As informações sensoriais para o controle postural. Fisioter Mov Curitiba 2006 abr/jun; 19(2): 11-8.

30. Thorbahn LDB, Newton RA. Use of the berg balance test to predict falls in elderly persons. Physical Therapy 1996 jun; 76(3): 576-85. 
